\title{
ATOMISTIC SIMULATION OF RADIATION-INDUCED AMORPHIZATION OF THE B2 ORDERED INTERMETALLIC COMPOUND NiTi*
}

\author{
Michael J. Sabochick \\ Air Force Institute of Technology \\ Dept. of Engineering Physics \\ Wright Patterson AFB, OH 45433 \\ Nghi Q. Lam \\ Materials Science Division \\ Argonne National Laboratory \\ Argonne, Il 60439
}

\section{December 1990}

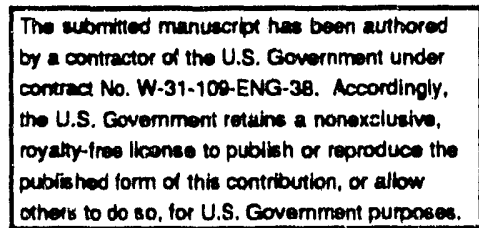

\section{DISCLAIMER}

\begin{abstract}
This report was prepared as an account of work sponsored by an agency of the United States Government. Neither the United States Government nor any agency thereof, nor any of their employees, makes any warranty, express or implied, or assumes any legal liability or responsibility for the accuracy, completeness, or usefulness of any information, apparatus, product, or process disclosed, or represents that its use would not infringe privately owned rights. Reference herein 10 any specific commercial product, process, or service by trade name, trademark, manufacturer, or otherwise does not necessarily constitute or imply its endorsement, recommendation, or favoring by the United States Government or any agency thereof. The views and opinions of authors expressed herein do not necessarily state or reflect those of the United States Government or any agency thereof.
\end{abstract}

Presented at the Materials Research Society Fall Meeting, Symposium A: Surface Chemistry and Beam-Solid Interactions; Symposium F: Kinetics of Phase Transformations, Nov. 26-30, 1990, Boston, MA.

*Work supported by the U. S. Department of Energy, BES-Materials Sciences, under Contract W-31-109-Eng-38 and from the Air Force Institute of Technology.

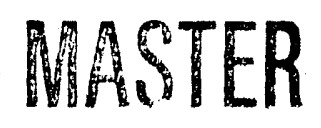




\title{
ATOMISTIC SIMULATION OF RADIATION-INDUCFD AMORPHIZATION OF THE B2 ORDERED INTERMETALLIC COMPOUND NiTi
}

\author{
MICHAEL J. SABOCHICK ${ }^{-1}$ and NGHI Q. LAM ${ }^{2}$
}

${ }^{1}$ Air Force Institute of Technology, Department of Engineering Physics,
Wright-Patterson Air Force Base, OH 45433-6583
${ }^{2}$ Argonne National Laboratory, Materials Science Division, Argonne, IL 60439

\section{ABSTRACT}

Amorphization of the B2 intermetallic compound NiTi under electron irradiation has been investigated using molecular dynamics. The effect of irradiation was simulated using two processes: 1) $\mathrm{Ni}$ and $\mathrm{Ti}$ atoms were exchanged, resulting in chemica! disorder, and 2) Frenkel pairs were introduced, leading to the formation of stable point. defects and also chemical disorder upon mutual recombination of interstitials ard vacancies. After $\sim 0.4$ exchanges per atom, the first process resulted in an energy increase of approximately $0.11 \mathrm{eV} /$ atom and a volume increase of $1.91 \%$. On the other hand, after introducing -0.5 Frenkel pairs per atom, the second process led to smaller increases of $0.092 \mathrm{eV} /$ atom in energy and $1.43 \%$ in volume. The calculated radial distribution functions (RDFs) were essentially identical to each other and to the calculated RDF of a quenched liquid. The structure factor, however, showed that long-range order was still present after atom exchanges, while the introduction of Frenkel pairs resulted in the loss of long-range order. It was concluded that point defects are necessary for amorphization to occur in $\mathrm{NiTi}$, although chemical disorder alone is capable of storing enough energy to make the transition possible.

\section{INTRODUCTION}

Computer simulation studies of amorphization induced by electron irradiation have concentrated on the question of what key factors are essential to induce the crystalline-to-amorphous (C-A) transition. In the earliest investigation, all of which were of pure systems, the introduction of point defects (either as single interstitials or as Frenkel pairs) resulted in amorphization [1-3]. Similar results were obtained upon the introduction of interstitials into a binary Lennard-Jones system [4]. Although few pure metals have been made amorphous experimentally, these simulations supported the importance of point defects in the amorphization process, in general agreement. with point-defect theories of amorphization [5-7]. In the first study of the amorphization of an intermetallic compound using realistic potentials, on the other hand, simply exchanging $\mathrm{Ni}$ and $\mathrm{Zr}$ atoms in simulated $\mathrm{NiZr}_{2}$ was sufficient to induce amorphization $[8,9]$, supporting the theory of amorphization based on chemical disorder [10]. In recent work on $\mathrm{Cu}_{\mathrm{m}} \mathrm{Ti}_{\mathrm{n}}$ compounds, the effects of chemical disorder and point defect introduction were studied in the same material. It was found that chemical disorder alone was insufficient to drive the crystalline-to-amorphous (C-A) transition, which occurred only after point defects were introduced $[11,12]$.

We report here the results of the simulation of $\mathrm{NiTi}$, a $\mathrm{B} 2$ compound which has been amorphized in experiments under electron irradiation. The purpose of this work was to further pursue the question of the relative importance of point defects versus chemical disorder with regard to amorphization through the study of another compound. In particular, we wish to shed light on the apparently contradictory conclusions found in the simulations of $\mathrm{NiZr}_{2}$ and $\mathrm{Cu}_{\mathrm{m}} \mathrm{Ti}_{\mathrm{n}}$ compounds. Interatomic potential 
- functions were derived for $\mathrm{NiTi}$, and the effects of both chemical disorder and Frenkel pair introduction were investigated using simulation methods similar to those of $\mathrm{Cu}_{\mathrm{m}} \mathrm{Ti}_{\mathrm{n}}$ study $[11,12]$.

\section{METHOD}

The computational methods employed in the present work were molecular statics and molecular dynamics, using a modified version of the code DYNAMO [13]. The simulations used a rectangular volume containing $N=1024$ atoms, the edge lengths of which were free to vary with external pressure.

The interatomic potential functions for $\mathrm{Ni}, \mathrm{Ti}$ and $\mathrm{Ni}$ - $\mathrm{Ti}$ were determined using a modification of the method of $\mathrm{Oh}$ and Johnson [14]. The potentials for $\mathrm{Ni}$ and $\mathrm{Ti}$ were fitted to the binding energy, lattice constants, elastic constants and vacancy formation energy of the pure metals. The potential for $\mathrm{Ni}$ - $\mathrm{Ti}$ was then determined by fitting the structure, lattice constants and heat of formation of the compound. The fitting procedure was identical to that described in [12]; the details for NiTi are discussed else where [15].

Chemical disorder was introduced by exchanging randomly-selected pairs of $\mathrm{Ni}$ and $\mathrm{Ti}$ atoms, as in [12]. Various system properties were then calculated as a function of the number of exchanges, i.e., increasing chemical disorder. Similarly, the effects of point defects were determined in a separate set of computer runs by calculating system properties as a function of the number of Frenkel pairs (FPs) introduced. In all cases, after a given number of atom exchanges or FP introductions, the systems were equilibrated at $160 \mathrm{~K}$ for 5000 time steps and properties calculated during an additional 1000 time steps. These properties were compared against those of the perfect crystal at the same temperature, and against those of a system that was quenched to $160 \mathrm{~K}$ from the liquid at $4000 \mathrm{~K}$.

\section{RESULTS}

The two most straightforward properties to calculate in simulations are the energy and volume. In Figures $1 \mathrm{a}$ and $1 \mathrm{~b}$, these properties are shown as a function of "dose" $D$. Here, $D$ is defined as either 1) the number of Ni-Ti pairs exchanged per atom, or 2) the number of FPs introduced per atom. As in the previous studies of $\mathrm{Cu}_{\mathrm{m}} \mathrm{Ti}_{\mathrm{n}}$ compounds [11,12], exchanging atoms and introducing Frenkel pairs were both found to increase the system energy and volume. In $\mathrm{NiTi}$, however, it was found that exchanging atoms led to a larger energy and volume increase than did FP introduction; in fact, the system energy and volume were larger than those of the quench.

The finding that the energies and volumes of the systems subjected to atom exchanges and to FP introduction are respectively larger and smaller than the queach values suggests that chemical disordering has induced the C-A transition, while FP introduction did not. The definition of "amorphous" however, is based on structure and not on energy or volume. Structure was investigated first by calculating the radial distribution function (RDF), which is shown for some of the systems in Fig. 2. Curves $\mathrm{B}$ and $\mathrm{E}$, showing the RDF after 1000 exchanges $(D=0.977)$ and of the quench, are rather similar, suggesting that atom exchanges lead to amorphization. Curves $C$ and $D$ show two stages during the introduction of FPs, after $100(D=0.0977)$ and 500 $(D=0.488)$ FPs. The RDF after $500 \mathrm{FP}$ is essentially indistinguishable from that of the quench.

A quantitative comparison of the RDFs of the treated systems with those of the perfect crystal and the quench was made possible by calculating a correlation coefficient 
Fig. 1. Energy, volume and correlation coefficient $G$ as a function of dose $D$.

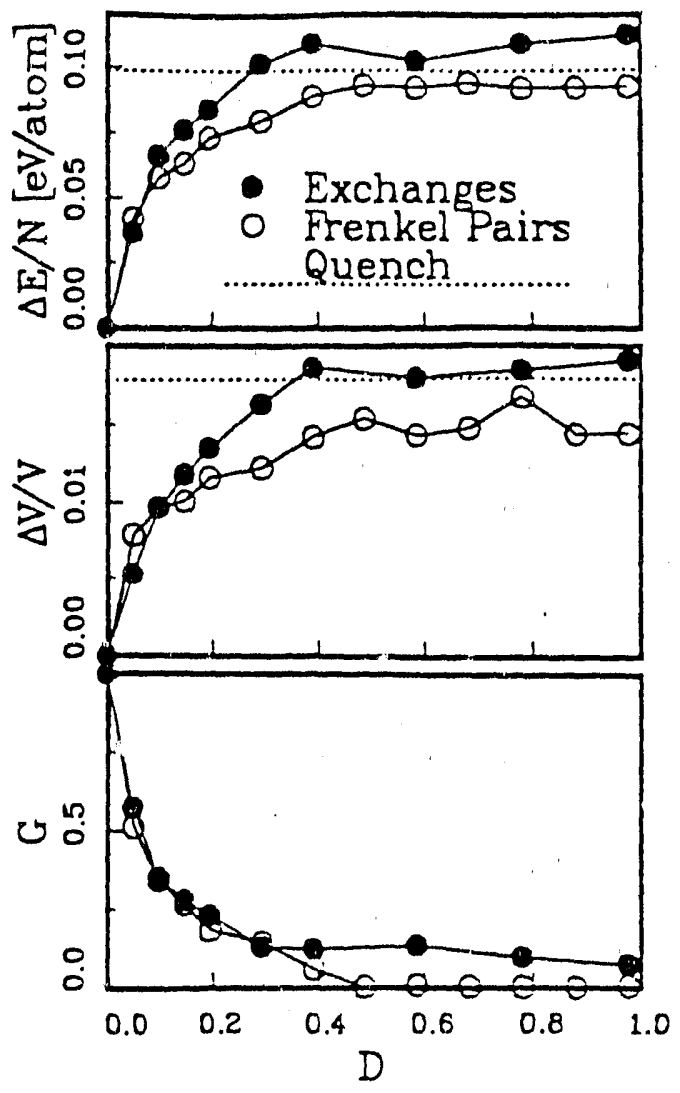

$G$. For a given RDF, $G$ has a value of 1 or 0 if the PDF is identical to that of the perfect crystal or the quench, respectively [12]. The calculated correlation coefficient is plotted as a function of $D$ in Fig. 1c. As was evident from a visual comparison of curves $D$ and $E$ in Fig. 2, $G$ is essentially zero after 500 FPs $(D=0.488)$. Atom exchanges led to $G \approx 0.1$ for $D=0.977$. For comparison, $G \approx 0.5$ was obtained after atom exchanges in $\mathrm{Cu}_{m} \mathrm{Ti}_{\mathrm{n}}$ compounds [12]. Evidently, atom exchanges resulted in larger lattice distortions in NiTi than they did in $\mathrm{Cu}_{m} \mathrm{Ti}_{n}$ compounds.

The results of the energy, volume and RDF calculations suggest that exchanging atoms (i.e., chemical disorder) may be suficient to amorphize NiTi. Although the RDF after atom exchanges is very similar to that of the quench (particularly in a visual comparison), they are not identical based on the correlation coefficient $G$. A more rigorous comparison of structure can be made by calculating the structure factor $S(\mathrm{k})$ given by

$$
S(\mathrm{k})=\frac{1}{N}\left|\sum_{j=1}^{N} \exp \left(i \mathrm{k} \cdot \mathbf{r}_{j}\right)\right|^{2}
$$

where $N$ is the number of atoms and $r_{j}$ is the position of atom $j$. The structure factor is shown for various systems and orientations of $\mathbf{k}$ in Fig. 3. A comparison of curves $C$ and $D$ in this Figure show's that the introduction of FPs led to a structure factor virtually identical to those of the quench, and a clear loss of long-range order. The structure factor after atom exchanges, however, clearly shows evidence of residual long-range order as many of the same peaks found in the perfect crystal remain after the exchanges. 
Fig. 2. Radial distribution function at 160K after the following treatments: A) perfect crystal; B) 1000 exchanges $[D=0.077] ; \quad C) 100$ FPs $[D=0.0977]$; D) 500 FPs $[D=0.488]$; and $E)$ quench from $4000 \mathrm{~K}$.
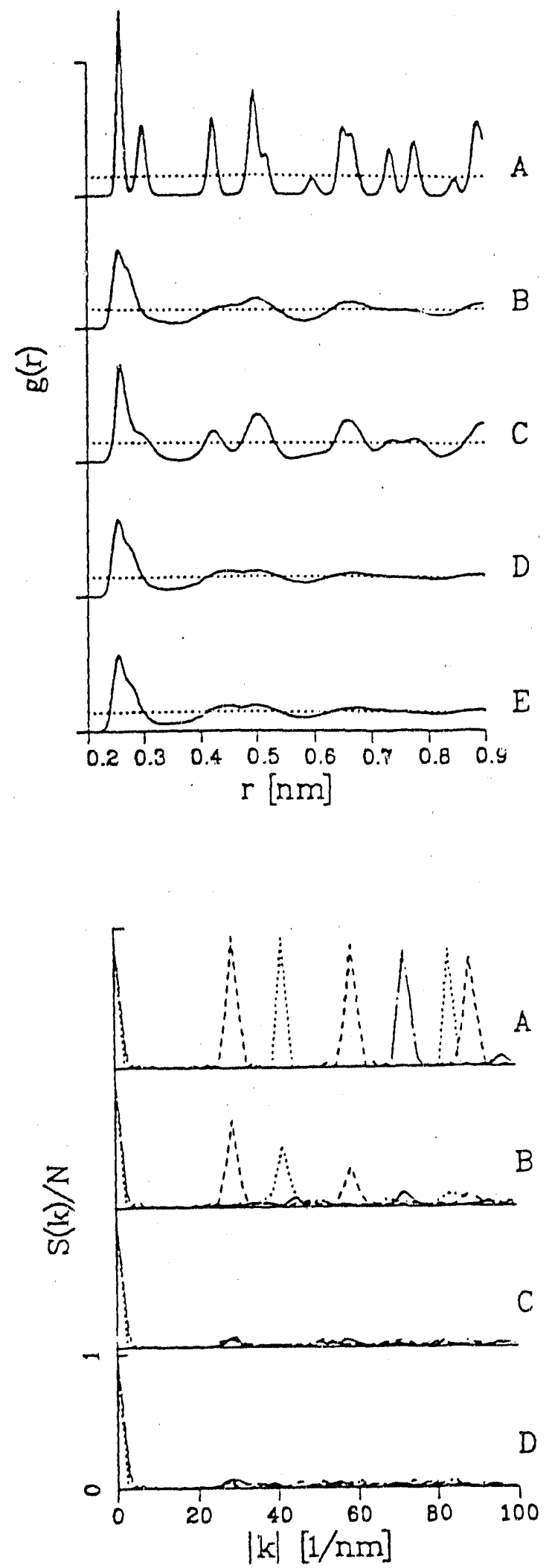

\section{DISCUSSION}

The results of the present work differ from the previous study of $\mathrm{Cu}_{m} \mathrm{Ti}_{D}[11,12]$ compounds in significant ways. In the $\mathrm{Cu}_{m} \mathrm{Ti}_{n}$ compounds, point defects were found to be necessary to induce the C-A transformation. Chemical disorder alone could not increase the energy of the system sufficiently to trigger the transition, because the energy of the chemically disordered state was intermediate between the crystalline and 
amorphous states. In $\mathrm{NiTi}$, however, the chemically disordered state has been found to have a higher energy than the amorphous state, as shown in Fig. 1. Thus, chemical disorder alone can store enough energy to drive the C-A transformation. Nevertheless, the chemically disordered state was apparently metastable since it was not observed to transform into the amorphous state.

The observation that chemical disorder leads to a fairly large energy increase in $\mathrm{NiTi}$ and a smaller increase in CuTi is explained by the antisite-pair energies of CuTi and $\mathrm{NiTi}$, which have been calculated to be $0.384 \mathrm{eV}$ and $1.015 \mathrm{eV}$, respectively [16-18]. The fact that the introduction of FPs leads to a lower system energy than does the exchange of $\mathrm{Ni}-\mathrm{Ti}$ pairs suggests that point defects actually reduce the energy of the chemically disordered state in NiTi.

The results of the present work on NiTi are similar in some ways to those found in the study of $\mathrm{NiZr}_{2}$ by Massobrio et al. $\{9\}$, in which it was concluded that chemicil disorder alone was sufficient to amorphize this compound. If that is true, chemical disorder alone must be able to increase the system energy such that the C-A transition is possible, as we have found in $\mathrm{NiTi}$. This transition may occur in $\mathrm{NiZr}_{2}$ because it has a complicated C16-type structure, as opposed to the simple B2 structure of NiTi.

The fact that the RDFs of chemically-disordered NiTi and amorphous NiTi are very similar, yet the structure factors are so different, indicates that the RDF is not very discriminating among various disordered states. In light of this finding, it would be interesting to calculate the structure factor for the chemically-disordered $\mathrm{NiZr}_{2}$ studied by Massobrio et al. [9]. It is possible that they may have observed a chemicallydisordered state that was not actually amorphous.

\section{CONCLUSION}

The amorphization of the intermetallic compound $\mathrm{NiTi}$ has been investigated using computer simulation. As in the previous study of $\mathrm{Cu}_{\mathrm{m}} \mathrm{Ti}_{\mathrm{n}}$ compounds, both atom exchanges and FP introduction resulted in energy and volume increases. In contrast to the $\mathrm{Cu}_{\mathrm{m}} \mathrm{Ti}_{n}$ compounds, however, atom exchanges led to larger increases than did FP introduction. Based on structure factor calculations, it was concluded that the chemically-disordered state was not amorphous but rather a higher-energy metastable state; FP introduction, on the other hand, did induce the C-A transition.

The results of the present work suggest that the importance of chemical disorder as a driving force for amorphization depends on the compound in question. In the case of the $\mathrm{Cu}_{\mathrm{m}} \mathrm{Ti}_{\mathrm{n}}$ compounds, point defects were required to introduce enough energy to drive the C-A transition, but chemical disorder alone was found to be sufficient in $\mathrm{NiTi}$ and probably in $\mathrm{NiZr}_{2}$. In either case, it is evident that a complete study on the computer, including the calculation of point defect and antisite properties as well as the simulation of the C-A transition, is essential to understand the key factors for amorphization in a given compound.

\section{ACKNOWLEDGEMENTS}

The authors would like to thank Drs. P.R. Okamoto and L. E. Rehn for many stimulating discussions. Part of this work was performed at Argonne National Laboratory while one of us (MJS) was a Faculty Research Participant supported by the Argcnne Division of Educational Programs. Support from the U.S. Department of Energy, BES-Materials Sciences, under Contract W-31-109-Eng-38, and from the Air Force Institute of Technology, is gratefully acknowledged. 
1. K. Maeda and S. Takeuchi, Phil. Mag. B 52, 955 (1985).

2. H. Hsieh and S. Yip, Phys. Rev. Lett. 59, 2760 (1987).

3. Y. Limoge, A. Rahman, H. Hsieh and S. Yip, J. Non-Cryst. Solids 99, 75 (1088).

4. H. Hsieh and S. Yip, Phys. Rev. B 39, 7476 (1989).

5. Y. Limoge and A. Barbu, Phys. Rev. B 30, 2212 (1984).

6. E. P. Simonen, Nucl. Instr. and Meth. B 16, 198 (1986).

7. D. F. Pedraza, J. Mater. Res. 1, 425 (1986).

8. C. Massobrio, V. Pontikis and G. Martin, Phys. Rev. Lett. 62, 1142 (1989).

9. C. Massobrio, V. Pontikis and G. Martin, Phys. Rev. B 41, 10486 (1990).

10. D. E. Luzzi and M. Meshii, Res Mechanica 21, 207 (1087).

11. M. J. Sabochick and N. Q. Lam, Scripta Met. et Mater. 24, 565 (1000).

12. M. J. Sabochick and N. Q. Lam, Phys. Rev. B, accepted for publication.

13. M. S. Daw, M. I. Baskes and S. M. Foiles (private communication).

14. D. J. Oh and R. A. Johnson, J. Mater. Res. 3, 471 (1988).

15. M. J. Sabochick and N. Q. Lam, to be published.

16. J. R. Shoemaker, D. Wesley, M. R. Wharton, M. L. Oehrli, M. J. Sabochick and N. Q. Lam, Mater. Res. Soc. Proc. 193, 330 (1990).

17. J. R. Shoemaker, R. T. Lutton, D. Wesley, W. R. Wharton, M. L. Oehrii, M. S. Herte, M. J. Sabochick and N. Q. Lam, J. Mater. Res., accepted for publication.

18. R. T. Lutton, M. J. Sabochick and N. Q. Lam, presented at the Mater. Res. Soc. Fall Meeting, Syposium K: Defects in Materials, Boston MA, Nov. 26-30, 1990. 

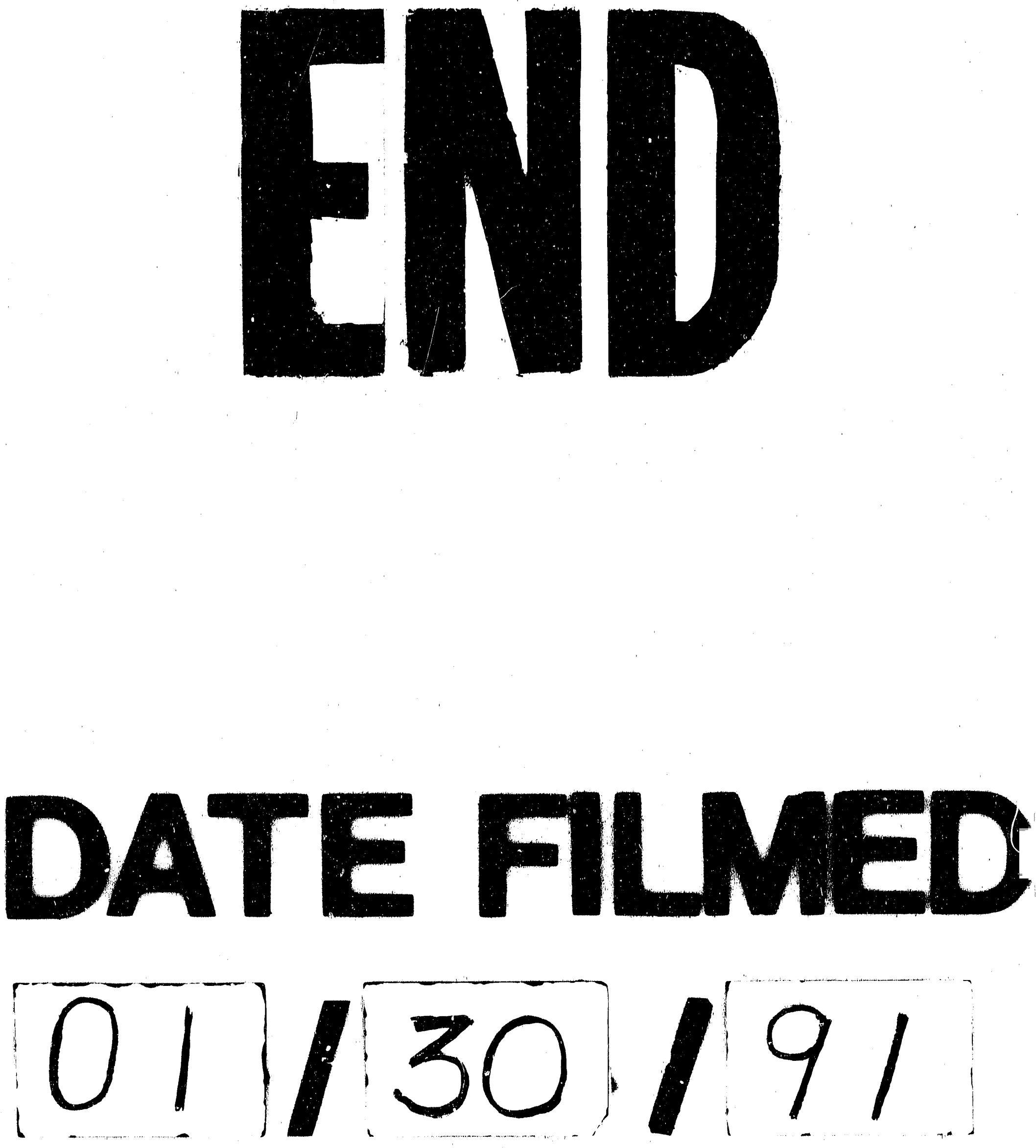
